\title{
BREAST CANCER AND YOUR RELATION WITH SEXUAL HORMONES
}

Gabriel Somma de Araújo1, Felipe Carrijo Montella1', Isabella Carneiro Oliveira Gonçalves Vaz', Isabella Reis Ristov¹, Vanessa Lara Guimarães', Danielle Brandão Nascimento'1

${ }^{1}$ Centro Universitário de Anápolis - UniEVANGÉLICA - Anápolis (GO), Brazil.

Sexual hormones, used primarily for pregnancy prevention and hormone replacement therapy (HRT), although they may help treat menstrual disorders and benign disorders of the uterus and ovary, are related to breast cancer. This neoplasm is the second major neoplasm in women, just losing for non-melanoma skin cancer. Besides that, facts that can possibly modulate the risk of breast cancer still being study to confirm their real influence in this pathology, especially the use of contraceptives and hormone replacement therapy. PubMed, Lilacs, SciELO, and Google Scholar databases were searched for studies from 2011 to 2018 that examined the association between sex hormones and breast cancer, as well as epidemiological numbers and other risk factors. The descriptors used to study the studies included: gonadal steroid hormones, breast neoplasms, risk factors, estrogens, and progesterone. A total of 22 studies were included in the review. Although most studies have shown a relationship between sex hormones and the incidence of breast cancer, in this study we conclude that the benefits of these hormones outweigh the risks of developing this type of cancer. In addition, it was concluded that other risk factors such as obesity, sedentarism, nulliparity, late gestation, family history, late menopause, early menarche, absence of lactogenesis, and advanced age influence the incidence of this pathology when compared to sex hormones. 